\title{
Papers
}

\section{Day care in infancy and risk of childhood acute lymphoblastic leukaemia: findings from UK case-control study}

\author{
C Gilham, J Peto, J Simpson, E Roman, T O B Eden, M F Greaves, F E Alexander, for the UKCCS Investigators
}

\begin{abstract}
Objective To test the hypothesis that reduced exposure to common infections in the first year of life increases the risk of developing acute lymphoblastic leukaemia.

Design and setting The United Kingdom childhood cancer study (UKCCS) is a large population based case-control study of childhood cancer across 10 regions of the UK.

Participants 6305 children (aged 2-14 years) without cancer; 3140 children with cancer (diagnosed 1991-6), of whom 1286 had acute lymphoblastic leukaemia (ALL).

Main outcome measure Day care and social activity during the first year of life were used as proxies for potential exposure to infection in infancy.

Results Increasing levels of social activity were associated with consistent reductions in risk of ALL; a dose-response trend was seen. When children whose mothers reported no regular activity outside the family were used as the reference group, odds ratios for increasing levels of activity were $0.73(95 \%$ confidence interval 0.62 to 0.87 ) for any social activity, 0.62 ( 0.51 to 0.75 ) for regular day care outside the home, and 0.48 (0.37 to 0.62 ) for formal day care (attendance at facility with at least four children at least twice a week) ( $\mathrm{P}$ value for trend $<0.001)$. Although not as striking, results for non-ALL malignancies showed a similar pattern ( $\mathrm{P}$ value for trend $<0.001)$. When children with non-ALL malignancies were taken as the reference group, a significant protective effect for ALL was seen only for formal day care (odds ratio $=0.69,0.51$ to $0.93 ; \mathrm{P}=0.02$ ). Similar results were obtained for $\mathrm{B}$ cell precursor common ALL and other subgroups, as well as for cases diagnosed above and below age 5 years.

Conclusion These results support the hypothesis that reduced exposure to infection in the first few months of life increases the risk of developing acute lymphoblastic leukaemia.
\end{abstract}

\section{Introduction}

The idea that infections are involved in the aetiology of childhood leukaemia dates back to the 1940 s. $^{1}$ Two key papers appeared in 1988. Greaves proposed that a deficit of exposure to infectious agents in infancy and subsequent "delayed" infectious challenge were causal factors in the development of B cell precursor common acute lymphoblastic leukaemia, ${ }^{2}$ which is responsible for the childhood peak of acute lymphoblastic leukaemia (ALL) at age 2-5 years. ${ }^{34}$ Kinlen proposed that population influx into isolated communities (population mixing) could generate excesses of childhood leukaemia by causing mini-epidemics of one or more infections to which leukaemia may be a rare response. ${ }^{5}$ The UK childhood cancer study
(UKCCS), a large population based case-control study, ${ }^{6}$ was designed to test several hypotheses, one of which was that leukaemias and lymphomas may be caused by abnormal responses to common infectious agents. Here, we focus on Greaves's hypothesis that immunological isolation in infancy increases the risk of $\mathrm{B}$ cell precursor common ALL (cALL). No single protective agent or transmission pathway has been identified, so proxy variables for exposure to infection must be used. The literature on infectious illnesses occurring in day care settings suggests that social interactions with other children outside the home may be important. ${ }^{7-9}$ Several studies of childhood leukaemia have used such proxies. ${ }^{10-19}$

Precise molecular subclassification of cALL is potentially important for these analyses. The two largest subgroups are those with hyperdiploidy (hyperdiploid ALL) and with fusion of the TEL and AML1 genes (TEL-AML1 ALL). Most (possibly all) children with these lesions have affected clones present at the time of birth, ${ }^{20}$ so initiation usually occurs in utero. However, the modest level of concordance in identical twins with one affected by cALL (approximately 10\%), together with the much greater frequency of these lesions in cord blood than the lifetime risk of the cALL subtype, ${ }^{22}$ indicates that at least one postnatal event also occurs in the development of cALL. Greaves's original hypothesis relates to the promotional factors that affect the frequency of this second event.

The UKCCS included all childhood cancers. ${ }^{6}$ In this paper we compare social activity of cases and controls during the first year of life for ALL and subgroups of ALL. We also compare ALL with non-ALL malignancies. We excluded children aged under 2 years at the time of diagnosis (cases) or pseudodiagnosis (controls) in order to avoid both dilution of results through overlap for younger children of the two time windows in which associations in opposite directions are predicted and the potential for early symptoms of leukaemia to influence attendance at day care.

\section{Methods \\ Participants}

This case-control study was conducted in 10 regions across the United Kingdom between 1991 and 1996. The UKCCS study design, data collection and consenting procedures, ethical approvals, and participation rates are described in detail elsewhere. ${ }^{63}$ Briefly, children diagnosed as having a confirmed malignancy were ascertained through paediatric oncology units, and two controls matched to each case for sex, month and year of birth, and region of residence at diagnosis were randomly selected from population registers. Age at diagnosis of the case was designated as the age at "pseudodiagnosis" of the matched control. A structured questionnaire was used to interview 
Table 1 Numbers of cases and controls overall (aged 2-14 years) and aged 2-5 at diagnosis or pseudodiagnosis

\begin{tabular}{lcc} 
Group or subgroup & Age 2-14 & Age 2-5 \\
\hline Controls & 6305 & 2497 \\
\hline Acute lymphoblastic leukaemia (ALL): & 1286 & 677 \\
\hline cALL & 798 & 475 \\
\hline TEL-AML1 & 139 & 82 \\
\hline Hyperdiploid ALL & 420 & 248 \\
\hline Non-ALL malignancies: & 1854 & 554 \\
\hline Central nervous system tumours & 578 & 157 \\
\hline Hodgkin's disease & 117 & 9 \\
\hline Other leukaemia* & 192 & 50 \\
\hline Non-Hodgkin's lymphoma & 227 & 49 \\
\hline Others $†$ & 740 & 289 \\
\hline All cases & 3140 & 1231
\end{tabular}

CALL=B cell precursor common ALL; TEL-AML1=ALL with fusion of the TEL and AML1 genes. *Leukaemias other than ALL.

†Malignancies other than leukaemias, lymphomas, and central nervous system tumours.

parents of 3838 cases and 7629 controls face to face. Questions about social activity focused on activity with other infants and children, and included information on the number of sessions a week and the number of children attending for specific activities before starting school.

\section{Exposure variables}

We defined "social activity" as regular activity (at least once a week) with other infants who were not members of the same household. We defined "day care" as attendance (at least once a week) at a day nursery, nursery school, play group, mother and toddler group, or childminder. We defined "formal day care" as any attendance at a day nursery or nursery school, at least two half day sessions a week at a playgroup or mother and toddler group, or at least two half day sessions a week at a childminder with a minimum of four children attending. We used a hierarchical variable based on these three exposures (social activity, day care, and formal day care) as an overall measure of social activity in the first year of life.

\section{Statistical analysis}

We excluded children given a diagnosis or pseudodiagnosis before the age of 2 years (649 cases and 1320 controls), as well as children with Down's syndrome (49 cases and 4 controls), which left 9445 eligible children (3140 cases and 6305 controls) (table 1). We analysed data for all cancers combined and separately for ALL, cALL, TEL-AML1 ALL, hyperdiploid ALL, and non-ALL malignancies. To increase precision, we compared each case subgroup with all controls. We also did a case-case comparison of ALL and cALL versus non-ALL malignancies. We used unconditional logistic regression procedures in Stata version 7 to estimate odds ratios and $95 \%$ confidence intervals. ${ }^{24}$ We used likelihood ratio tests to assess associations. We assessed trends across the combined hierarchical variable by treating it as a continuous variable.

\section{Results}

Most (86\%) mothers of controls reported some social activity with children outside the family in the first year of life (table 2). Any such activity was associated with a reduced risk of ALL (odds ratio $=0.66 ; \mathrm{P}<0.001$ ). The risk ratios for cALL alone and for the cytogenetic subgroups TEL-AML1 and hyperdiploidy were similarly reduced. Analyses of non-ALL malignancies combined gave a similar result; the only individually statistically significantly reduced risk was for the largest group-central nervous system tumours. With respect to the case-case comparison (ALL $v$ non-ALL malignancies), the dichotomous variable "any social activity" was not significantly reduced. Analyses restricted to 2-5 year olds produced similar results (table 2), but we found no evidence that the association was stronger in the childhood peak (2-5 years) than at older ages.

Each category of malignancy showed a significant inverse trend as level of social activity increased (table 2). The statistically significant trend $(\mathrm{P}=0.04)$ for the comparison of ALL with nonALL malignancies (right hand column) is due largely to the reduced odds ratio for formal day care (odds ratio $=0.69,95 \%$ confidence interval 0.51 to 0.93 ). Analyses restricted to cases aged 2-5 years gave similar results, although statistical significance was reduced.

The proportion of children who had an older sibling living in the home at the time of birth was similar for ALL (56\%), cALL (54\%), non-ALL malignancies (57\%), and controls (57\%), and we observed no significant trends with numbers of older siblings in any diagnostic group (table 3). As any relation between social activity and ALL might be expected to be more marked among children born into households without other children, we repeated the analyses in table 2 for children with and without older siblings. The odds ratio for formal day care was $0.61(0.42$ to 0.87$)$ for ALL in children without older siblings and $0.38(0.26$ to 0.54 ) for those with older siblings, a non-significant difference in the opposite direction to that anticipated.

Estimated risks for children starting day care in the first year of life showed no marked trends with age at first attendance (table 4). The greatest reduction in risk of ALL, however, was seen in children who attended formal day care during the first three months of life, for whom the odds ratio remained statistically significant when we used non-ALL malignancies as the reference group (odds ratio $=0.52,0.32$ to $0.83 ; \mathrm{P}=0.007$ ).

\section{Discussion}

The UKCCS was a large, nationwide, population based investigation into the causes of childhood cancer. One of the principal hypotheses tested was that immunological isolation in infancy increases the risk of cALL. In this report we assess immunological isolation indirectly, mainly by lack of social activity as indicated by day care attendance in the first year of life. The overall results for ALL show a consistent and statistically significant reduction in risk for each level of social activity in the first year of life and a dose-response trend across increasing levels of activity. Results were similar for cALL and other ALL subgroups, although the numbers for TEL-AML1 ALL were small. The findings were similar when we restricted the analysis to children aged 2-5 years at diagnosis. However, because we also saw similar trends for non-ALL malignancies, we repeated the analyses for ALL with non-ALL malignancies as the comparison group. The estimated risk for formal day care attendance in the first year of life remained significant (odds ratio $=0.69,95 \%$ confidence interval 0.51 to $0.93 ; \mathrm{P}=0.02$ ); the effect was most marked for formal day care within the first three months of life (odds ratio $=0.52,0.32$ to $0.83 ; \mathrm{P}=0.007$ ).

\section{Potential limitations}

Studies of this type, in which participants are recruited after their disease status is known and information on exposure is obtained retrospectively by questionnaire, are susceptible to several well documented biases. ${ }^{63} 24$ Participants who respond may differ from those who do not; some responses may systematically differ between cases and controls; and behavioural variables, such as 
Table 2 Levels of social activity in the first year of life for acute lymphoblastic leukaemia (ALL), ALL subgroups, and non-ALL malignancies

\begin{tabular}{|c|c|c|c|c|c|c|c|c|c|c|c|c|}
\hline \multirow[b]{2}{*}{ Activity level } & \multirow[b]{2}{*}{$\begin{array}{l}\text { Controls } \\
\text { No (\%) }\end{array}$} & \multicolumn{2}{|r|}{ ALL } & \multicolumn{2}{|c|}{ CALL } & \multicolumn{2}{|c|}{ TEL-AML1 } & \multicolumn{2}{|c|}{ Hyperdiploid ALL } & \multicolumn{2}{|c|}{ Non-ALL malignancies } & \multirow{2}{*}{$\begin{array}{c}\text { ALL } v \text { non-ALL } \\
\text { malignancy: } \\
\text { odds ratio* } \\
(95 \% \text { CI) }\end{array}$} \\
\hline & & No (\%) & $\begin{array}{c}\text { Odds ratio* } \\
(95 \% \mathrm{CI})\end{array}$ & № (\%) & $\begin{array}{c}\text { Odds ratio* } \\
(95 \% \mathrm{Cl})\end{array}$ & No $(\%)$ & $\begin{array}{c}\text { Odds ratio* } \\
(95 \% \mathrm{Cl})\end{array}$ & № $(\%)$ & $\begin{array}{c}\text { Odds ratio* } \\
(95 \% \mathrm{CI})\end{array}$ & No $(\%)$ & $\begin{array}{c}\text { Odds ratio* } \\
(95 \% \mathrm{CI})\end{array}$ & \\
\hline \multicolumn{13}{|c|}{ Aged over 2 years } \\
\hline$\overline{\text { Total No† }}$ & 6238 & \multicolumn{2}{|r|}{1272} & \multicolumn{2}{|r|}{791} & \multicolumn{2}{|r|}{138} & \multicolumn{2}{|r|}{417} & \multicolumn{2}{|r|}{1825} & \\
\hline $\begin{array}{c}\text { Any social } \\
\text { activity }\end{array}$ & $\begin{array}{l}5343 \\
(85.7)\end{array}$ & $\begin{array}{l}1020 \\
(80.2)\end{array}$ & $\begin{array}{c}0.66 \\
(0.56 \text { to } 0.77)\end{array}$ & $\begin{array}{c}640 \\
(80.9)\end{array}$ & $\begin{array}{c}0.67 \\
\text { (0.55 to } 0.82 \text { ) }\end{array}$ & $\begin{array}{c}110 \\
(79.7)\end{array}$ & $\begin{array}{c}0.59 \\
(0.38 \text { to } 0.90)\end{array}$ & $\begin{array}{l}335 \\
(80.3)\end{array}$ & $\begin{array}{c}0.64 \\
(0.50 \text { to } 0.83)\end{array}$ & $\begin{array}{l}1496 \\
(82.0)\end{array}$ & $\begin{array}{c}0.78 \\
(0.68 \text { to } 0.90)\end{array}$ & $\begin{array}{c}0.88 \\
(0.73 \text { to } 1.06)\end{array}$ \\
\hline $\begin{array}{r}\text { No social } \\
\text { activity }\end{array}$ & $895(14.4)$ & $\begin{array}{c}252 \\
(19.8)\end{array}$ & 1.00 & $\begin{array}{c}151 \\
(19.1) \\
\end{array}$ & 1.00 & $\begin{array}{c}28 \\
(20.3)\end{array}$ & 1.00 & $\begin{array}{c}82 \\
(19.7) \\
\end{array}$ & 1.00 & $\begin{array}{c}329 \\
(18.0)\end{array}$ & 1.00 & 1.00 \\
\hline $\begin{array}{l}\text { Social } \\
\text { activity, } \\
\text { but no day } \\
\text { care }\end{array}$ & $\begin{array}{l}2840 \\
(45.5)\end{array}$ & $\begin{array}{c}587 \\
(46.1)\end{array}$ & $\begin{array}{c}0.73 \\
(0.62 \text { to } 0.87)\end{array}$ & $\begin{array}{l}358 \\
(45.3)\end{array}$ & $\begin{array}{c}0.74 \\
(0.60 \text { to } 0.91)\end{array}$ & $\begin{array}{c}60 \\
(43.5)\end{array}$ & $\begin{array}{c}0.61 \\
(0.38 \text { to } 0.97)\end{array}$ & $\begin{array}{c}199 \\
(47.7)\end{array}$ & $\begin{array}{c}0.76 \\
(0.58 \text { to } 1.00)\end{array}$ & $\begin{array}{c}880 \\
(48.2)\end{array}$ & $\begin{array}{c}0.83 \\
(0.71 \text { to } 0.96)\end{array}$ & $\begin{array}{c}0.91 \\
(0.74 \text { to } 1.11)\end{array}$ \\
\hline $\begin{array}{l}\text { Informal day } \\
\text { care only }\end{array}$ & $\begin{array}{l}1768 \\
(28.3)\end{array}$ & $\begin{array}{c}325 \\
(25.6)\end{array}$ & $\begin{array}{c}0.62 \\
(0.51 \text { to } 0.75)\end{array}$ & $\begin{array}{l}218 \\
(27.6)\end{array}$ & $\begin{array}{c}0.67 \\
\text { (0.53 to } 0.84 \text { ) }\end{array}$ & $\begin{array}{c}38 \\
(27.5)\end{array}$ & $\begin{array}{c}0.60 \\
(0.36 \text { to } 1.00)\end{array}$ & $\begin{array}{c}105 \\
(25.2)\end{array}$ & $\begin{array}{c}0.57 \\
(0.42 \text { to } 0.78)\end{array}$ & $\begin{array}{c}435 \\
(23.8)\end{array}$ & $\begin{array}{c}0.72 \\
(0.61 \text { to } 0.85)\end{array}$ & $\begin{array}{c}0.90 \\
(0.71 \text { to } 1.13)\end{array}$ \\
\hline $\begin{array}{l}\text { Formal day } \\
\text { care }\end{array}$ & $735(11.8)$ & $\begin{array}{l}108 \\
(8.5)\end{array}$ & $\begin{array}{c}0.48 \\
(0.37 \text { to } 0.62)\end{array}$ & $64(8.1)$ & $\begin{array}{c}0.44 \\
(0.32 \text { to } 0.60)\end{array}$ & $12(8.7)$ & $\begin{array}{c}0.47 \\
(0.24 \text { to } 0.94)\end{array}$ & $31(7.4)$ & $\begin{array}{c}0.38 \\
(0.24 \text { to } 0.59)\end{array}$ & $\begin{array}{l}181 \\
(9.9)\end{array}$ & $\begin{array}{c}0.73 \\
(0.59 \text { to } 0.90)\end{array}$ & $\begin{array}{c}0.69 \\
(0.51 \text { to } 0.93)\end{array}$ \\
\hline $\mathrm{P}$ for trendf & & & $<0.001$ & & $<0.001$ & & 0.04 & & $<0.001$ & & $<0.001$ & 0.04 \\
\hline \multicolumn{13}{|c|}{ Aged 2-5 years } \\
\hline Total No† & 2475 & \multicolumn{2}{|r|}{671} & \multicolumn{2}{|r|}{471} & \multicolumn{2}{|r|}{81} & \multicolumn{2}{|r|}{248} & \multicolumn{2}{|r|}{546} & \\
\hline $\begin{array}{c}\text { Any social } \\
\text { activity }\end{array}$ & $\begin{array}{l}2156 \\
(87.1) \\
\end{array}$ & $\begin{array}{c}545 \\
(81.2) \\
\end{array}$ & $\begin{array}{c}0.63 \\
(0.50 \text { to } 0.79)\end{array}$ & $\begin{array}{c}387 \\
(82.2) \\
\end{array}$ & $\begin{array}{c}0.67 \\
\text { (0.51 to } 0.88) \\
\end{array}$ & $\begin{array}{c}68 \\
(84.0)\end{array}$ & $\begin{array}{c}0.73 \\
\text { (0.39 to } 1.35) \\
\end{array}$ & $\begin{array}{c}198 \\
(79.8) \\
\end{array}$ & $\begin{array}{c}0.57 \\
(0.40 \text { to } 0.80)\end{array}$ & $\begin{array}{c}447 \\
(81.9)\end{array}$ & $\begin{array}{c}0.66 \\
\text { (0.51 to } 0.85 \text { ) }\end{array}$ & $\begin{array}{c}0.98 \\
(0.73 \text { to } 1.31)\end{array}$ \\
\hline $\begin{array}{r}\text { No social } \\
\text { activity }\end{array}$ & $319(12.9)$ & $\begin{array}{c}126 \\
(18.8)\end{array}$ & 1.00 & $\begin{array}{c}84 \\
(17.8)\end{array}$ & 1.00 & $\begin{array}{c}13 \\
(16.0)\end{array}$ & 1.00 & $\begin{array}{c}50 \\
(20.2)\end{array}$ & 1.00 & $\begin{array}{c}99 \\
(18.1)\end{array}$ & 1.00 & 1.00 \\
\hline $\begin{array}{l}\text { Social } \\
\text { activity, } \\
\text { but no day } \\
\text { care }\end{array}$ & $\begin{array}{l}1023 \\
(41.3)\end{array}$ & $\begin{array}{c}294 \\
(43.8)\end{array}$ & $\begin{array}{c}0.70 \\
(0.54 \text { to } 0.89)\end{array}$ & $\begin{array}{c}195 \\
(41.4)\end{array}$ & $\begin{array}{c}0.69 \\
(0.52 \text { to } 0.93)\end{array}$ & $\begin{array}{c}35 \\
(43.2)\end{array}$ & $\begin{array}{c}0.74 \\
\text { (0.38 to } 1.43 \text { ) }\end{array}$ & $\begin{array}{c}115 \\
(46.4)\end{array}$ & $\begin{array}{c}0.69 \\
(0.48 \text { to } 0.99)\end{array}$ & $\begin{array}{c}234 \\
(42.9)\end{array}$ & $\begin{array}{c}0.70 \\
\text { (0.53 to 0.91) }\end{array}$ & $\begin{array}{c}1.03 \\
(0.75 \text { to } 1.42)\end{array}$ \\
\hline $\begin{array}{l}\text { Informal day } \\
\text { care only }\end{array}$ & 778 (31.4) & $\begin{array}{c}182 \\
(27.1)\end{array}$ & $\begin{array}{c}0.59 \\
(0.45 \text { to } 0.77)\end{array}$ & $\begin{array}{c}142 \\
(30.1)\end{array}$ & $\begin{array}{c}0.71 \\
(0.52 \text { to } 0.96) \\
\end{array}$ & $\begin{array}{c}25 \\
(30.9) \\
\end{array}$ & $\begin{array}{c}0.76 \\
\text { (0.38 to 1.53) }\end{array}$ & $\begin{array}{c}63 \\
(25.4) \\
\end{array}$ & $\begin{array}{c}0.51 \\
(0.34 \text { to } 0.76)\end{array}$ & $\begin{array}{c}139 \\
(25.5)\end{array}$ & $\begin{array}{c}0.59 \\
\text { (0.44 to } 0.79)\end{array}$ & $\begin{array}{c}1.01 \\
(0.71 \text { to } 1.43)\end{array}$ \\
\hline $\begin{array}{l}\text { Formal day } \\
\text { care }\end{array}$ & $355(14.3)$ & $\begin{array}{c}69 \\
(10.3)\end{array}$ & $\begin{array}{c}0.49 \\
(0.35 \text { to } 0.68)\end{array}$ & $\begin{array}{c}50 \\
(10.6)\end{array}$ & $\begin{array}{c}0.52 \\
(0.35 \text { to } 0.77)\end{array}$ & $8(9.9)$ & $\begin{array}{c}0.62 \\
(0.25 \text { to } 1.53)\end{array}$ & $20(8.1)$ & $\begin{array}{c}0.33 \\
(0.19 \text { to } 0.58)\end{array}$ & $\begin{array}{c}74 \\
(13.6)\end{array}$ & $\begin{array}{c}0.68 \\
(0.48 \text { to } 0.96)\end{array}$ & $\begin{array}{c}0.73 \\
(0.48 \text { to } 1.13)\end{array}$ \\
\hline$\overline{P \text { for trendf }}$ & & & $<0.001$ & & 0.004 & & 0.4 & & $<0.001$ & & 0.01 & 0.2 \\
\hline
\end{tabular}

CALL=B cell precursor common ALL; TEL-AML1=ALL with fusion of the TEL and AML1 genes.

${ }^{\star}$ Odds ratio for cases compared with all controls, or with non-ALL malignancies where stated, adjusted for age at diagnosis/pseudodiagnosis, sex, region, maternal age, mother working at time of birth, and deprivation.

tExcluding missing values.

‡Trend test across categories none through to formal day care.

Table 3 Number of older children in household ("siblings") at time of index birth for acute lymphoblastic leukaemia (ALL), ALL subgroups, and non-ALL malignancies

\begin{tabular}{|c|c|c|c|c|c|c|c|c|c|c|c|}
\hline \multirow[b]{2}{*}{$\begin{array}{l}\text { No of } \\
\text { siblings }\end{array}$} & \multirow[b]{2}{*}{$\begin{array}{c}\text { Controls } \\
\text { No (\%) }\end{array}$} & \multicolumn{2}{|r|}{ ALL } & \multicolumn{2}{|c|}{ CALL } & \multicolumn{2}{|c|}{ TEL-AML1 } & \multicolumn{2}{|c|}{ Hyperdiploid ALL } & \multicolumn{2}{|c|}{ Non-ALL malignancies } \\
\hline & & No (\%) & $\begin{array}{c}\text { Odds ratio }{ }^{\star} \\
(95 \% \mathrm{Cl})\end{array}$ & No $(\%)$ & $\begin{array}{c}\text { Odds ratio* } \\
(95 \% \mathrm{CI})\end{array}$ & No $(\%)$ & $\begin{array}{c}\text { Odds ratio* } \\
\text { (95\% CI) }\end{array}$ & No (\%) & $\begin{array}{c}\text { Odds ratio* } \\
(95 \% \text { CI) }\end{array}$ & No (\%) & $\begin{array}{c}\text { Odds ratio* } \\
(95 \% \mathrm{Cl})\end{array}$ \\
\hline \multicolumn{12}{|c|}{ Cases aged over 2 years } \\
\hline Total No† & 6197 & \multicolumn{2}{|c|}{1270} & \multicolumn{2}{|r|}{789} & \multicolumn{2}{|r|}{138} & \multicolumn{2}{|r|}{416} & \multicolumn{2}{|c|}{1830} \\
\hline None & $\begin{array}{l}2690 \\
(43.4)\end{array}$ & $\begin{array}{c}565 \\
(44.5)\end{array}$ & 1.00 & $\begin{array}{c}365 \\
(46.3)\end{array}$ & 1.00 & $62(44.9)$ & 1.00 & $\begin{array}{c}189 \\
(45.4)\end{array}$ & 1.00 & $\begin{array}{c}799 \\
(43.7)\end{array}$ & 1.00 \\
\hline 1 & $\begin{array}{l}2216 \\
(35.8)\end{array}$ & $\begin{array}{c}443 \\
(34.9)\end{array}$ & $\begin{array}{c}0.96 \\
\text { (0.84 to } 1.11)\end{array}$ & $\begin{array}{c}266 \\
(33.7)\end{array}$ & $\begin{array}{c}0.88 \\
(0.74 \text { to } 1.05)\end{array}$ & $49(35.5)$ & $\begin{array}{c}0.89 \\
(0.60 \text { to } 1.32)\end{array}$ & $\begin{array}{c}149 \\
(35.8)\end{array}$ & $\begin{array}{c}0.97 \\
(0.77 \text { to } 1.22)\end{array}$ & $\begin{array}{c}667 \\
(36.5)\end{array}$ & $\begin{array}{c}1.03 \\
(0.91 \text { to } 1.16)\end{array}$ \\
\hline 2 & $\begin{array}{c}899 \\
(14.5)\end{array}$ & $\begin{array}{c}182 \\
(14.3)\end{array}$ & $\begin{array}{c}0.99 \\
(0.82 \text { to } 1.21)\end{array}$ & $\begin{array}{c}115 \\
(14.6)\end{array}$ & $\begin{array}{c}0.94 \\
(0.74 \text { to } 1.20)\end{array}$ & $20(14.5)$ & $\begin{array}{c}0.86 \\
(0.50 \text { to } 1.48)\end{array}$ & 57 (13.7) & $\begin{array}{c}0.94 \\
(0.68 \text { to } 1.30)\end{array}$ & $\begin{array}{l}281 \\
(15.4)\end{array}$ & $\begin{array}{c}1.11 \\
(0.94 \text { to } 1.31)\end{array}$ \\
\hline$\geq 3$ & $392(6.3)$ & $80(6.3)$ & $\begin{array}{c}0.99 \\
(0.74 \text { to } 1.30)\end{array}$ & $43(5.4)$ & $\begin{array}{c}0.78 \\
\text { (0.54 to } 1.12)\end{array}$ & $7(5.1)$ & $\begin{array}{c}0.70 \\
(0.30 \text { to } 1.62)\end{array}$ & $21(5.0)$ & $\begin{array}{c}0.75 \\
\text { (0.46 to } 1.25 \text { ) }\end{array}$ & $83(4.5)$ & $\begin{array}{c}0.76 \\
(0.59 \text { to } 1.00)\end{array}$ \\
\hline P for trendf & & & 0.9 & & 0.2 & & 0.4 & & 0.4 & & 0.7 \\
\hline \multicolumn{12}{|c|}{ Cases aged 2-5 years } \\
\hline Total† & 2463 & \multicolumn{2}{|r|}{673} & \multicolumn{2}{|r|}{473} & \multicolumn{2}{|r|}{82} & \multicolumn{2}{|r|}{248} & \multicolumn{2}{|c|}{548} \\
\hline None & $\begin{array}{l}1071 \\
(43.5)\end{array}$ & $\begin{array}{c}308 \\
(45.8)\end{array}$ & 1.00 & $\begin{array}{c}221 \\
(46.7)\end{array}$ & 1.00 & $40(48.8)$ & 1.00 & $\begin{array}{c}114 \\
(46.0)\end{array}$ & 1.00 & $\begin{array}{c}256 \\
(46.7)\end{array}$ & 1.00 \\
\hline 1 & $\begin{array}{c}873 \\
(35.4)\end{array}$ & $\begin{array}{c}233 \\
(34.6)\end{array}$ & $\begin{array}{c}0.95 \\
(0.78 \text { to } 1.16)\end{array}$ & $\begin{array}{c}164 \\
(34.7)\end{array}$ & $\begin{array}{c}0.93 \\
(0.74 \text { to } 1.17)\end{array}$ & $29(35.4)$ & $\begin{array}{c}0.81 \\
(0.49 \text { to } 1.36)\end{array}$ & $89(35.9)$ & $\begin{array}{c}0.98 \\
(0.72 \text { to } 1.33)\end{array}$ & $\begin{array}{c}186 \\
(33.9)\end{array}$ & $\begin{array}{c}0.95 \\
(0.77 \text { to } 1.19)\end{array}$ \\
\hline 2 & $\begin{array}{c}366 \\
(14.9)\end{array}$ & $96(14.3)$ & $\begin{array}{c}0.95 \\
(0.72 \text { to } 1.25)\end{array}$ & $65(13.7)$ & $\begin{array}{c}0.89 \\
\text { (0.65 to } 1.22)\end{array}$ & $8(9.8)$ & $\begin{array}{c}0.52 \\
(0.23 \text { to } 1.16)\end{array}$ & $35(14.1)$ & $\begin{array}{c}0.97 \\
\text { (0.64 to 1.48) }\end{array}$ & $81(14.8)$ & $\begin{array}{c}1.05 \\
(0.78 \text { to } 1.40)\end{array}$ \\
\hline$\geq 3$ & $153(6.2)$ & $36(5.3)$ & $\begin{array}{c}0.85 \\
\text { (0.56 to } 1.29)\end{array}$ & $23(4.9)$ & $\begin{array}{c}0.72 \\
(0.44 \text { to } 1.19)\end{array}$ & $5(6.1)$ & $\begin{array}{c}0.81 \\
\text { (0.29 to } 2.25)\end{array}$ & $10(4.0)$ & $\begin{array}{c}0.61 \\
(0.29 \text { to } 1.27)\end{array}$ & $25(4.6)$ & $\begin{array}{c}0.80 \\
(0.50 \text { to } 1.28)\end{array}$ \\
\hline $\mathrm{P}$ for trend & & & 0.5 & & 0.2 & & 0.2 & & 0.4 & & 0.7 \\
\hline
\end{tabular}

CALL=B cell precursor common ALL; TEL-AML1=ALL with fusion of the TEL and AML1 genes.

${ }^{\star}$ Odds ratio for cases compared with all controls, adjusted for age at diagnosis/pseudo diagnosis, sex, region, maternal age, mother working at time of birth, and deprivation.

†Excluding missing values.

†Trend test across categories none through to $\geq 3$. 
Table 4 Effect of age at first day care during the first year of life for acute lymphoblastic leukaemia (ALL) and non-ALL malignancies

\begin{tabular}{|c|c|c|c|c|c|c|c|c|c|c|c|c|}
\hline \multirow{3}{*}{$\begin{array}{l}\text { Age first } \\
\text { attended } \\
\text { (months) }\end{array}$} & \multicolumn{6}{|c|}{ Day care } & \multicolumn{6}{|c|}{ Formal day care } \\
\hline & \multicolumn{3}{|c|}{ No (\%) } & \multicolumn{3}{|c|}{ Odds ratio* $(95 \% \mathrm{Cl})$} & \multicolumn{3}{|c|}{ No (\%) } & \multicolumn{3}{|c|}{ Odds ratio* $(95 \% \mathrm{Cl})$} \\
\hline & $\begin{array}{c}\text { Controls } \\
(n=6250) \dagger\end{array}$ & $\begin{array}{c}\text { ALL } \\
(n=1274) \dagger\end{array}$ & $\begin{array}{c}\text { Non-ALL } \\
(\mathrm{n}=1830) \dagger\end{array}$ & $\begin{array}{c}\text { ALL } v \\
\text { controls }\end{array}$ & $\begin{array}{c}\text { Non-ALL } v \\
\text { controls }\end{array}$ & $\begin{array}{c}\text { ALL } v \\
\text { non-ALL }\end{array}$ & $\begin{array}{c}\text { Controls } \\
(n=6269) \dagger\end{array}$ & $\begin{array}{c}\text { ALL } \\
(n=1278) \dagger\end{array}$ & $\begin{array}{c}\text { Non-ALL } \\
(n=1833) \dagger\end{array}$ & $\begin{array}{c}\text { ALL } v \\
\text { controls }\end{array}$ & $\begin{array}{c}\text { Non-ALL } v \\
\text { controls }\end{array}$ & $\begin{array}{c}\text { ALL } v \\
\text { non-ALL }\end{array}$ \\
\hline None & $\begin{array}{l}3736 \\
(59.8)\end{array}$ & $839(65.9)$ & $\begin{array}{l}1210 \\
(66.1)\end{array}$ & 1.00 & 1.00 & 1.00 & $\begin{array}{l}5534 \\
(88.3)\end{array}$ & $\begin{array}{l}1170 \\
(91.5)\end{array}$ & $\begin{array}{l}1652 \\
(90.1)\end{array}$ & 1.00 & 1.00 & 1.00 \\
\hline$<3$ & $\begin{array}{l}1091 \\
(17.5)\end{array}$ & $185(14.5)$ & $289(15.8)$ & $\begin{array}{c}0.71 \\
(0.60 \text { to } 0.85)\end{array}$ & $\begin{array}{c}0.88 \\
(0.76 \text { to } 1.02)\end{array}$ & $\begin{array}{c}0.82 \\
(0.66 \text { to } 1.02)\end{array}$ & $231(3.7)$ & $27(2.1)$ & $71(3.9)$ & $\begin{array}{c}0.56 \\
(0.37 \text { to } 0.83)\end{array}$ & $\begin{array}{c}1.07 \\
(0.81 \text { to } 1.40)\end{array}$ & $\begin{array}{c}0.52 \\
(0.32 \text { to } 0.83)\end{array}$ \\
\hline $3-5$ & 548 (8.8) & $94(7.4)$ & $130(7.1)$ & $\begin{array}{c}0.71 \\
\text { (0.56 to } 0.90)\end{array}$ & $\begin{array}{c}0.83 \\
(0.68 \text { to } 1.02)\end{array}$ & $\begin{array}{c}0.91 \\
\text { (0.68 to } 1.22)\end{array}$ & 204 (3.3) & $35(2.7)$ & $42(2.3)$ & $\begin{array}{c}0.71 \\
\text { (0.49 to } 1.03)\end{array}$ & $\begin{array}{c}0.77 \\
\text { (0.55 to } 1.08)\end{array}$ & $\begin{array}{c}1.01 \\
(0.62 \text { to } 1.64)\end{array}$ \\
\hline $6-11$ & 875 (14.0) & $156(12.2)$ & $201(11.0)$ & $\begin{array}{c}0.76 \\
(0.63 \text { to } 0.92)\end{array}$ & $\begin{array}{c}0.76 \\
(0.64 \text { to } 0.91)\end{array}$ & $\begin{array}{c}1.00 \\
(0.79 \text { to } 1.27)\end{array}$ & $300(4.8)$ & 46 (3.6) & 68 (3.7) & $\begin{array}{c}0.69 \\
(0.50 \text { to } 0.96)\end{array}$ & $\begin{array}{c}0.82 \\
(0.62 \text { to } 1.08)\end{array}$ & $\begin{array}{c}0.81 \\
(0.54 \text { to } 1.21)\end{array}$ \\
\hline
\end{tabular}

*Odds ratio for cases compared with all controls or with non-ALL malignancies where stated, adjusted for age at diagnosis or pseudodiagnosis, sex, region, maternal age, mother working at time of birth, and deprivation.

†Excluding missing values.

social activity outside the home, may be affected by the pre-clinical effects of incipient disease.

Some systematic differences between cases and controls existed in this study. Analysis of census data revealed that controls who agreed to take part were living in more affluent areas, ${ }^{623}$ and some control parents were interviewed when their children were older than their matched cases. The average interval from diagnosis or pseudodiagnosis to interview was six months for cases and 14 months for controls. ${ }^{6}$ Children destined to develop a malignancy may also have more periods of ill health in early life, leading to lower attendance at day care. Health status in early life will be the subject of a future paper from the UKCCS, but preliminary analyses (Roman, personal communication) indicate that, compared with controls, more frequent periods of illness are seen in children who develop solid tumours but not in children who develop ALL. If this is an effect rather than a cause of the development of cancer, reverse causation might contribute to the protective effect of day care for non-ALL malignancies but not for ALL.

Interpretation of our findings depends crucially on whether the protective effect of social activity for non-ALL malignancies is real or due to bias, as the protective effect for ALL is both smaller and less significant when non-ALL malignancies are used as the reference group. Despite this uncertainty, we believe that the difference between ALL and non-ALL malignancies may well be real. A prior hypothesis was that the risk of leukaemia would be increased by a lack of early social activity, and the effect of day care is particularly marked during the first three months of life ( $\mathrm{P}=0.007$ for ALL $v$ non-ALL malignancies), as was seen in a recent French study that compared children with ALL with children without cancer. ${ }^{16}$

Our data for "any social activity" are, inevitably, subjective and could have been affected by demographic factors including parental age and socioeconomic status. At interview, parents were offered a range of activities to describe groups that their children might have attended; they were also asked about the number of children present and number of sessions their child attended each week. We used the responses to these questions to derive the variables used in our analyses; the baseline was the children of those parents who reported no regular activity outside the home in the first year of life $-18.6 \%$ of parents of cases and $14.4 \%$ of parents of controls. We investigated the possibility of under-reporting by combining the two lowest categories in the combined exposure variable, and this did not affect the results.

\section{Comparison with other studies}

Other case-control studies of childhood leukaemia have looked at social activity and day care. ${ }^{10-19}$ Diversity exists for both ages at diagnosis and ages of day care attendance, as well as the definition of day care used. The only study that quantified exposure to other children reported a significant protective effect. ${ }^{18}$ Most other studies suggest a reduction in risk of around 30-40\% for day care attendance or social activity, though lack of statistical power often leads to imprecise risk estimates.

The difficulty of establishing small effects reliably is illustrated by the lack of consensus among studies investigating an association between childhood ALL and birth order or mother's parity. Although reduced risks in children with several older siblings have been seen in some studies, most studies, like ours, have found no such effects. ${ }^{25}$ As well as sibship position, other studies have considered different proxies for exposure to the spectrum of infectious agents. The only European study with comparable numbers of ALL cases to our series inferred social contact from parents' employment status and found no association. ${ }^{26}$ Several investigators have reported reduced risks of ALL or cALL in children with many infections, ${ }^{1526}$ or with specific infections in infancy, such as frequent otitis media or roseola, ${ }^{10} 12$ but others have not found such associations. ${ }^{17} 27$

In support of an infectious aetiology for childhood ALL, several ecological studies have reported that marked influxes of population into isolated areas are followed by transiently increased rates of childhood leukaemia. ${ }^{58}$ Furthermore, evidence of inherited susceptibility to ALL associated with HLA and alleles of other immune system genes is consistent with the suggestion that infection may be associated with ALL. The UKCCS has recently reported statistically significant associations between cALL and specific HLA-DPB1 variants. ${ }^{29}$ This is further supported by evidence that immunisation of infants may protect against ALL. ${ }^{27}$ 30-32

\section{Possible mechanisms}

The hypotheses proposed by Greaves and Kinlen differ with respect to their speculation as to the underlying mechanisms and the roles of specific infections, viral or otherwise, as well as the postulated timing of key events. ${ }^{25}$ Kinlen has proposed that the mini-epidemics that generate excesses of childhood leukaemia are due to one or a small number of specific though unknown leukaemia causing agents, probably viruses. For Greaves, however, the consequences of immunological isolation in the first year of life were predicted to be, firstly, inadequate priming of the naïve immune system and, secondly, continuing susceptibility to infections responsible for a later challenge, which, in the absence of adequate priming may precipitate a highly dysregulated immune response. This, in turn, was predicted to promote the development of cALL indirectly by proliferative stress to the bone marrow, which facilitated further mutations. The effect of the later infectious challenge is thus immunological 


\section{What is already known on this topic}

Childhood leukaemia is a biologically diverse disease and is likely to arise by several aetiological pathways

The common, B cell precursor, form of acute lymphoblastic leukaemia accounts for the incidence peak between 2 and 5 years of age, and immunological isolation may be a causal factor

Children attending day care have an increased risk of contracting a variety of common infections

\section{What this study adds}

Children attending day care centres on a regular basis in the first few months of life are less likely to develop acute lymphoblastic leukaemia than are children who do not

rather than leukaemogenic. Our study has examined the question of exposures in infancy by using social activity outside the home as proxy but does not contribute to the debate on underlying biological mechanisms.

\section{Conclusion}

Our results provide further support that social activity with other infants and children during the first few months of life protects against subsequent risk of ALL. The effect is less pronounced among cases diagnosed at age 2-5 years than at older ages and is not confined to cALL. The most plausible interpretation is that this protection comes from exposure to common infections. Similar associations have been reported for type 1 diabetes and allergies in children. ${ }^{33}{ }^{34}$ Whether early exposure to one or more specific infections, or to a spectrum of non-specific agents, protects against each of these disparate diseases remains to be clarified. Nevertheless, we conclude that some degree of early exposure to infection seems to be important for child health.

We thank the members of the UK Childhood Cancer Study Group for their support. We also thank local hospital staff, general practitioners, general practice staff, and UKCCS interviewers and technicians. We especially thank the families of the children included in the study. The UK childhood cancer study is sponsored and administered by the Leukaemia Research Fund. This study was conducted by 12 teams of investigators ( 10 clinical and epidemiological and two biological) based in university departments, research institutes, and the NHS in Scotland. The work was coordinated by a management committee. It was supported by the UK Children's Cancer Study Group of paediatric oncologists and by the National Radiological Protection Board.

Contributors: FEA, TOBE, MFG, JP, ER, and JS were involved in the design and conduct of the study throughout. FEA, CG, JP, and JS were responsible for management and analysis of the data. All authors contributed to writing the manuscript. ER is the guarantor.

Funding: Financial support has been provided by the Cancer Research Campaign and Imperial Cancer Research Fund (now Cancer Research UK), the Leukaemia Research Fund, and the Medical Research Council through grants to their units; by the Leukaemia Research Fund, the Department of Health, the Electricity Association, the Irish Electricity Supply Board, the National Grid Company, and Westlakes Research (Trading) through grants for the general expenses of the study; and by the Kay Kendall Leukaemia Fund for the associated laboratories studies. The investigation in Scotland is funded by the Scottish Office, Scottish Power, Scottish Hydro-Electric, and Scottish Nuclear.

Competing interests: None declared.

Ethical approval: See previous publications (references 6 and 23).

1 Cooke J. The incidence of acute leukemia in children. JAMA 1942;119:547-50.

2 Greaves MF. Speculations on the cause of childhood acute lymphoblastic leukemia. Leukemia 1988;2:120-5.
3 Greaves MF, Alexander FE. An infectious etiology for common acute lymphoblastic leukemia in childhood? Leukemia 1993;7:349-60.

4 Greaves MF. Aetiology of acute leukaemia. Lancet 1997;349:344-9.

5 Kinlen LJ. The longitudinal study and the social distribution of cancer. BMJ 1988;297:1070.

6 UK Childhood Cancer Study Investigators. The United Kingdom childhood cancer study: objectives, materials and methods. Br J Cancer 2000;82:1073-102.

7 Fleming DW, Cochi SL, Hightower AW, Broome CV. Childhood upper respiratory tract infections: to what degree is incidence affected by day-care attendance? Pediatric 1987;79:55-60.

8 Louhiala PJ, Jaakkola N, Ruotsalainen R, Jaakkola JJ. Form of day care and respiratory infections among Finnish children. Am J Public Health 1995;85:1109-12.

9 Rasmussen F, Sundelin C. Use of medical care and antibiotics among preschool children in different day care settings. Acta Paediatr Scand 1990;79:838-46.

10 Chan LC, Lam TH, Li CK, Lau YL, Li CK, Yuen HL, et al. Is the timing of exposure to infection a major determinant of acute lymphoblastic leukaemia in Hong Kong? Paediatr Perinat Epidemiol 2002;16:154-65.

11 Dockerty JD, Skegg DCG, Elwood JM, Herbison GP, Becroft DMO, Lewis ME. Infections, vaccinations, and the risk of childhood leukaemia. $\mathrm{Br} J$ Cancer 1999;80:1483-9.

12 Neglia JP, Linet MS, Shu XO, Severson RK, Potter JD, Mertens AC, et al. Patterns of infection and day care utilization and risk of childhood acute lymphoblastic leukaemia. Br J Cancer 2000;82:234-40.

13 Petridou E, Kassimos D, Kalmanti M, Kosmidis H, Haidas S, Flytzani V, et al. Age of exposure to infections and risk of childhood leukaemia. BMJ 1993;307:774.

14 Infante-Rivard C, Fortier I, Olson E. Markers of infection, breast-feeding and childhood acute lymphoblastic leukaemia. Br J Cancer 2000;83:1559-64.

15 Perrillat F, Clavel J, Auclerc MF, Baruchel A, Leverger G, Nelken B, et al. Day-care, early common infections and childhood acute leukaemia: a multicentre French case-control study. Br J Cancer 2002;86:1064-9.

16 Jourdan-Da Silva N, Perel Y, Mechinaud F, Plouvier E, Gandemer V, Lutz P, et al. Infectious diseases in the first year of life, perinatal characteristics and childhood acute leukaemia. BrJ Cancer 2004;90:139-45.

17 Petridou E, Trichopoulos D, Kalapothaki V, Pourtsidis A, Kogevinas M, Kalmanti M, et al. The risk profile of childhood leukaemia in Greece: a nationwide case-control study. Br J Cancer 1997;76:1241-7.

18 Ma X, Buffler PA, Selvin S, Matthay KK, Wiencke JK, Wiemels JL, et al. Daycare attendance and risk of childhood acute lymphoblastic leukaemia. Br J Cancer 2002;86:141924.

19 Rosenbaum PF, Buck GM, Brecher ML. Early child-care and preschool experiences and the risk of childhood acute lymphoblastic leukemia. Am J Epidemiol 2000;152:1136-44

20 Wiemels JL, Cazzaniga G, Daniotti M, Eden OB, Addison GM, Masera G, et al. Prenatal origin of acute lymphoblastic leukaemia in children. Lancet 1999;354:1499-503.

21 Greaves MF, Wiemels J. Origins of chromosome translocations in childhood leukaemia. Nat Rev Cancer 2003:3:639-49.

22 Mori H, Colman SM, Xiao Z, Ford AM, Healy LE, Donaldson C, et al. Chromosome translocations and covert leukemic clones are generated during normal fetal development. Proc Natl Acad Sci USA 2002;99:8242-7.

23 Law GR, Smith AG, Roman E, UK Childhood Cancer Study Investigators. The importance of full participation: lessons from a national case-control study. Br J Cancer 2002;86:350-5.

24 Breslow NE, Day NE. Statistical methods in cancer research. Vol 1. The analysis of case-control studies. Lyon: International Agency for Research on Cancer, 1980.

25 Roman E, Simpson J, Ansell P, Lightfoot T, Mitchell CD, Eden OB. Perinatal and reproductive factors: a report on haematological malignancies from the UKCCS. Eur J Can cer 2005 ; in press.

26 Steensel-Moll HA, Valkenburg HA, van Zanen GE. Childhood leukemia and infectious diseases in the first year of life: a register-based case-control study. Am J Epidemiol 1986;124:590-4.

27 Schuz J, Kaletsch U, Meinert R, Kaatsch P, Michaelis J. Association of childhood leukaemia with factors related to the immune system. Br J Cancer 1999:80:585-90.

28 Kinlen LJ. Epidemiological evidence for an infective basis in childhood leukaemia. $\mathrm{Br} J$ Cancer 1995; 71:1-5.

29 Taylor GM, Dearden S, Ravetto P, Ayres M, Watson P, Hussain A, et al. Genetic susceptibility to childhood common acute lymphoblastic leukaemia is associated with polymorphic peptide-binding pocket profiles in HLA-DPB $1 * 0201$. Hum Mol Genet 2002;11:1585-97.

30 Groves FD, Gridley G, Wacholder S, Shu XO, Robison LL, Neglia JP, et al. Infant vaccinations and risk of childhood acute lymphoblastic leukaemia in the USA. Br J Cancer 1999;81:175-8.

31 Auvinen A, Hakulinen T, Groves F. Haemophilus influenzae type B vaccination and risk of childhood leukaemia in a vaccine trial in Finland. Br J Cancer 2000;83:956-8.

32 Groves F, Sinha D, Auvinen A. Haemophilus influenzae type b vaccine formulation and risk of childhood leukaemia. Br J Cancer 2002;87:511-2.

33 McKinney PA, Okasha M, Parslow RC, Law GR, Gurney KA, Williams R, et al. Early social mixing and childhood type 1 diabetes mellitus: a case-control study in Yorkshire, UK. Diabet Med 2000;17:236-42.

34 Kramer U, Heinrich J, Wjst M, Wichmann HE. Age of entry to day nursery and allergy in later childhood. Lancet 1999;353:450-4.

(Accepted 15 March 2005)

doi 10.1136/bmj.38428.521042.8F

Cancer Research UK Epidemiology and Genetics Unit, Institute of Cancer

Research, Sutton SM2 5NG

C Gilham statistician

Department of Epidemiology and Population Health, London School of Hygiene and Tropical Medicine, London WC1E 7HT

J Peto professor of epidemiology

Leukaemia Research Fund Epidemiology and Genetics Unit, Department of 
Health Sciences, University of York, York YO10 5DD

J Simpson research fellow

E Roman professor of epidemiology

Academic Unit of Paediatric Oncology, Christie Hospital and Central Manchester and Manchester Children's University Hospitals NHS Trusts, Manchester M20 $4 \mathrm{BX}$

T O B Eden professor of paediatric oncology
Section of Haemato Oncology, Institute of Cancer Research, London SW3 6JB M F Greaves professor of cell biology

Public Health Sciences, University of Edinburgh, Edinburgh EH8 9AG

$\mathrm{F}$ E Alexander professor of statistics

Correspondence to: T O B Eden tim.eden@manchester.ac.uk 